\title{
Enseñanza de la historia e identidad nacional: Un vínculo a historizar desde la experiencia chilena, 1850-1930
}

\author{
The teaching of history and national identity: \\ Historicizing its links from the Chilean \\ experience, 1850-1930
}

\author{
L'enseignement de l'histoire et l'identité \\ nationale : Historiciser les liens à partir de \\ I'expérience chilienne, 1850-1930
}

\author{
Sol Serrano \\ Pontificia Universidad Católica de Chile
}

RESUMEN

La historia de la educación y la didáctica de la historia suelen sostener que la enseñanza de la historia y los ritos cívicos fueron forjadores de la identidad nacional y de los nacionalismos a partir de la formación de los estados nacionales en el siglo XIX. Este artículo intenta historizar este supuesto en base al estudio del caso de Chile entre 1850-1930 incorporando variables como clase social, genero, etnia, asentamiento territorial, demografía con sus distintos ritmos y periodificaciones. Concluye que la historia nacional fue marginal en relación a la historia europea y que fue minoritaria dentro del currículo; que "lo" nacional se incluye tardíamente y transversalmente en el currículo; que los alumnos que estudiaron historia fueron una franca minoría dentro de una ya baja cobertura escolar. Muestra los debates ideológicos sobre la historia nacional en los textos escolares y los ritos cívicos en la vida escolar concluyendo que fueron "desde arriba" y minoritarios en relación a los ritos y actividades propias de los establecimientos. Concluye, asimismo, que la identidad de la comunidad escolar y de la comunidad local fueron predominantes y que sin ella, el estudio de la "identidad nacional" resulta abstracta y lineal.

Descriptores: historia de la educación en Chile, enseñanza de la historia, identidad nacional en Chile. 


\section{ABSTRACT}

In history of education and in history education (the teaching of history), it is often said that national identities and nationalisms were built through the teaching of history and the civic rites, beginning from the times of the building of nation-states in the nineteenth century. This article attempts to historicize this assumption by analyzing the case of Chile between 1850 and 1930, while incorporating variables such as social class, gender, ethnicity, territorial settlements, demographic rhythms, and periodization. It concludes that the national was not dominant in the curriculum and was marginal in relation to the European history, the national was introduced quite late and in a transversal way, and the students taking the courses constituted a minority in a context characterized by low school coverage. It discusses the presence of ideological debates on national history in the textbooks, which was minor, and the civic rites in the school life, which were not many in relation to rites and activities pertaining to schooling and "desde arriba." It concludes that the identity of the school community and the local community played a major role and without considering this, the study of the "national identity" may be rendered abstract and lineal.

Key words: history of education in Chile, teaching of history, national identity in Chile.

\section{RÉSUMÉ}

En histoire de l'éducation et dans l'enseignement de l'histoire, on dit souvent que les identités nationales et les nationalismes ont été construits par l'enseignement de l'histoire et les rites civiques à partir des temps où les états nationaux ont été érigés au dix-neuvième siècle. Cet article tente d'historiciser cette hypothèse en analysant le cas du Chili entre 1850 et 1930, tout en y incorporant des variables comme les classes sociales, les sexes, l'ethnicité, les accords territoriaux, les rythmes démographiques et la périodisation. Nous concluons que le national n'était pas dominant, mais marginal, dans les programmes d'enseignement, relativement à l'histoire de l'Europe; le national a été introduit plutôt tardivement et de façon transversale; les étudiants qui suivirent ces cours étaient en minorité dans un contexte caractérisé par une faible couverture scolaire. L'article examine le fait qu'il y avait peu de débats idéologiques sur l'histoire nationale dans les manuels scolaires et de rites civiques dans la vie courante à l'école, à comparer avec les rites et les activités se rapportant à l'instruction et "desde arriba.» Nous concluons que l'identité de la communauté scolaire et la communauté locale jouèrent un rôle prépondérant et qu'en ne prenant pas ce fait en considération, l'étude de l'identité nationale risque de devenir abstraite et linéale.

Mots clés: histoire de l'éducation au Chili, l'enseignement de I'histoire, l'identité nationale au Chili.

\section{Introducción}

T a relación entre la enseñanza de la historia y la formación de la identidad _nacional o de los nacionalismos es un campo de estudio que se ha desarrollado con fuerza a partir de los debates ya clásicos sobre el nacionalismo en la década del 80 (Anderson, 1993; Gellner, 1983; Hobsbawm, 1991). Tanto en la historia de la educación (Del Pozo, 2008; Herrera, 2008; Lowe, 1999) como en la didáctica de la historia (Barton, 2012, p. 92; Carretero \& López, 2012; Riekenberg, 1991; Symcox \& Wilschut, 2009) se suele afirmar que la disciplina histórica surgida en el siglo XIX y la introducción de su enseñanza en el currículo escolar habrían tenido por objeto y habrían sido forjadoras de la identidad nacional y de los nacionalismos. Este supuesto adolece de un peligro tautológico, es decir, se refiere solo a la dimensión nacional sin 
situarla en el conjunto de la enseñanza y de las prácticas escolares. Este artículo tiene por objeto contribuir a historizar estos vínculos con el objeto de rescatar su complejidad. La evidencia está tomada del caso de Chile aproximadamente entre 1850 y 1930. El caso es particular, pero no excepcional. Son los problemas los que interesan y estos pueden aplicarse a una multiplicidad de experiencias La investigación sobre un caso específico permite hacerse preguntas comunes, que es lo que este artículo quisiera aportar.

El primer tema es calibrar la enseñanza de la historia nacional o patria en el conjunto de la enseñanza de la historia y del currículo dentro en una línea de tiempo; el segundo, es preguntarse quienes estudian historia efectivamente y la relación que ello tiene con la estructura social del sistema educacional, sus tramas demográficas y territoriales. El tercero es el uso de manuales de historia nacional que expresan no solo un nacionalismo supuestamente unificador sino los debates por la hegemonía política e ideológica y los conflictos internos entre quienes toman las decisiones sobre lo que se estudia. Y finalmente, el artículo trata los ritos cívicos en el conjunto de las prácticas y ritos escolares. El artículo concluye que la enseñanza de la historia fue universalista más que nacional; que lo "nacional" se incorpora hacia 1930 transversalmente en el currículo. Concluye que los estudiantes de historia fueron una minoría por la cobertura general y que se restringió prácticamente a la educación secundaria urbana. Muestra finalmente que los ritos cívicos de carácter nacionalista impuestos por el Estado fueron menos relevante, menos vivos y congregantes de la comunidad escolar que sus propias celebraciones y actividades. La pertenencia a la comunidad escolar, a la comunidad local se desarrolla como una nueva sociabilidad que genera a su vez nuevas pertenencia, entre ella, la nacional. ${ }^{1}$

\section{La relevancia de la historia nacional}

La enseñanza de la historia no entro a los estudios colegiales de la mano de las grandes narrativas nacionales, como suele indicarse, sino antes, y su primer objeto no fue la formación de una identidad nacional sino la formación moral de sus hombres dirigentes. Tal como en la enseñanza clásica y humanista, no era la historicidad la que interesaba, era el presente, la historia como "maestra de la vida" (Garcia \& Deluc, 2004; Wilschut, 2012). Con la Revolución Francesa la enseñanza de la historia adquirió un sentido más propiamente político, sin por ello abandonar su carácter ejemplarizante como la exaltación del republicanismo romano. En América Latina, y específicamente en Chile, también predominaron los estudios clásicos luego de las Independencias pues era la enseñanza que formaba ciudadanos virtuosos (Cruz, 2002; Jaksic, 1989; Tanck, 2011). En aquellos países donde el paso de monarquía a republica fue por medios revolucionarios, esa formación se hizo tanto más pertinente pues la legitimidad del poder político se había desplazado de lo religioso a lo jurídico y desde Dios a los ciudadanos. La historia fue auxiliar de los estudios clásicos hasta que a mediados del siglo XIX ingreso como asignatura en los colegios. En Chile (cuya educación colegial o secundaria se denominaba liceo) se siguió de cerca el caso francés y se estudió con textos franceses traducidos y adaptados localmente. Así, se estudiaba historia antigua (incorporaba Oriente y Grecia) y romana con el 
texto de Victor Boreau, medieval con el de Lame Fleury e historia moderna con el compendio de Jules Michelet, publicado en 1827 y traducido por Andrés Bello en 1848. El texto de Michelet, L'Histoire Moderne. Introduction a L'Histoire Universelle, es evidentemente una historia de Europa, especialmente de Europa Occidental, aunque incorpora lo que llama Europa del Norte y Oriente. La periodización va desde la toma de Constantinopla por los turcos hasta la Revolución Francesa. Es una historia militar, política, comercial y también de las letras y de las artes. En sus 600 páginas, Francia ocupa un espacio equilibrado en relación al resto de las regiones según el periodo. Bello redujo considerablemente su extensión, pero mantuvo el equilibrio de la obra. (Michelet, 1860, 1897). La "historia de América y Chile" ocupaba 3 de las 13 horas semanales, se impartía en el último año y no requería textos propio sino "la recopilación del profesor" (MMJCIP, 1859). ${ }^{2}$ Los alumnos que llegaban a al último año eran pocos ya fuera por deserción o porque varios liceos de provincia impartían solo los tres primeros años de humanidades.

Un ínfima parte de los estudiantes cursaba historia nacional, la misma que pertenecía a la elite gobernante y letrada que empezaba a escribir y a leer las primeras narrativas históricas nacionales (Gazmuri, 2006). Los primeros historiadores fueron en su mayoría profesores del Instituto Nacional, ministros de estado o parlamentarios. Posiblemente por ello no se requería texto. Se aprendía en la catedra, en la prensa, en el debate parlamentario, en las publicaciones locales, en la vida familiar y social. Era parte de la política, del nuevo espacio público masculino. En cambio Cicerón sí debía estudiarse en el colegio.

El discurso político del periodo le asigno a la educación una función eminentemente ciudadana segmentada socialmente. La elite debía formarse en la virtud pública desarrollada, como se dijo, por los estudios clásicos y por la historia de la "civilización.” La escuela primaria para los sectores populares tenía por objeto formar a los futuros ciudadanos alfabetos e industriosos que tendrían derecho a voto. Las niñas populares también debían ser educadas no por derecho propio sino en cuanto serían las madres de los futuros ciudadanos. En un país cuya población rural comprendía un $70 \%$ de la población de acuerdo al Censo de 1861, alfabetizar significaba el primer peldaño hacia el ingreso a la civilización. Es en el concepto de civilización donde se incorpora el concepto de nación entendida por la elite gobernante como la máxima expresión de la misma. Hasta entrado el siglo XIX la escuela era civilizatoria y su lucha era contra la ignorancia. Si la identidad se define por la representación que se hace de sí mismo como miembro de una comunidad en oposición a otra, la identidad nueva que a nivel discursivo se buscaba formar - al margen de sus logros - era la pertenencia de la "nación" a la "civilización" en oposición a la "barbarie." Por ello el texto de Domingo Faustino Sarmiento llamando a civilizar a la barbarie, escrito en Chile en 1842, el mismo año en que organizaba la instrucción primaria, sintetizo el discurso letrado de su tiempo (Sarmiento, 1845).

\section{Quienes estudian la historia nacional}

La relación causal entre enseñanza de la historia, identidad nacional y nacionalismo suele obviar a los actores concretos, sus diferenciaciones sociales, territoriales y 
también cronológicas. El concepto de "identidad nacional” ha dejado atrás su antiguo carácter esencialista e inmutable comprendiéndose como una construcción histórica. Aun así, aparece como un concepto globalizante que comprendería al conjunto de la sociedad organizada como estado nacional sin dar cuenta de que la "pertenencia nacional" es un proceso social diferenciado. Si la enseñanza de la historia genera una "identidad nacional," al menos cabría preguntarse quienes accedieron a esa enseñanza y a qué ritmo. La cobertura de la educación colegial fue baja en el siglo XIX en la mayoría de los países occidentales. En Chile, 17 de cada mil jóvenes de ambos sexos entre 10 y 20 años estaban matriculados en un liceo en 1885 . Hacia 1900 solo un $3 \%$ de quienes ingresaban a la educación colegial la terminaba. Esos eran los que estudiaban historia patria. En la escuela primaria, que educaba masivamente a las clases populares y donde se supone que se urde la relación a que aludimos, la cobertura y su composición es crucial. En Noruega 3\% de los niños recibía alguna enseñanza de la historia en 1853 (Svein, 2001, p. 111); un $46 \%$ de la primaria en Francia en 1888 y un $85 \%$ a comienzos del siglo XX (Garcia \& Leduc, 2004, p. 116). En Chile bordeaban el 2\% hacia fines de siglo XIX.

El estado chileno manifestó su preocupación por la enseñanza de la historia y la geografía nacional haciéndolas asignaturas obligatorias a partir de la Ley de Instrucción Primaria (1860). A partir de ella, la Escuela Normal de preceptores (1842) y la de preceptoras (1853) separo la asignatura de historia de Chile de las "Nociones generales de historia y particulares de Chile." Se incorporó el estudio de la Constitución, único ramo de la formación general que diferenciaba a la masculina de la femenina (Cox \& Gysling, 2009, p. 73). Un tercio de los preceptores en el cambio de siglo eran normalistas. La cobertura de la primaria creció de un $11 \%$ en 1858 a un $22 \%$ en 1895. De estos, un 2\% terminaba la escuela, es decir, cursaba el último año en el que se enseñaba historia de Chile. Debido a los altos índices de inasistencia y deserción, en 1882 la asignatura se incorporó a todos los grados de la primaria y se introdujo la educación cívica. Hacia 1910 un 52\% de los escolares de la primaria asistían a un curso de historia de Chile y 20\% asistía al ramo de educación cívica (AE, 1909). ${ }^{3}$ Otro indicador de la enseñanza de la historia nacional es la circulación de los textos de estudio. Aunque la información es parcial, se puede afirmar que solo un 5\% de los textos escolares impresos por el gobierno en 1854 y 1862 correspondió a historia de Chile (MMJCIP, 1854, 1862). Es presumible que el contacto de estos niños con "lo nacional" no estuviera en la enseñanza de la historia sino en los silabarios y libros de lectura que tenían alusiones e ilustraciones del país, en los mapas que entonces empezaron a ser colgados en las paredes.

Las escuelas fiscales de niñas se fundaron simultáneamente a las de niños, pero con una matrícula inicial muy menor que se igualo al terminar el siglo (MJCIP, 1899). Distinto fue el caso en la educación secundaria. Los primeros colegios femeninos a mediados del XIX eran privados y de corta vida, o colegios de elite fundados por religiosas francesas. Aun así, las materias de estudios no eran tan distintas en la formación general a la masculina pública. Estudiaban historia antigua, medieval y moderna europea, así como historia de Chile y América. Se diferenciaba por agregar historia eclesiástica y sagrada (de La Taille, 2012, p. 245). El Estado no fundó liceos 
femeninos hasta avanzado el siglo, en 1890, por considerar que dicha educación correspondía a la familia y no al Estado. Sin embargo, el conflicto ideológico en torno a la secularización hizo temer que la educación femenina de élite exclusivamente católica significara un menoscabo y retroceso del estado docente. En efecto, la demanda por educación femenina pública y gratuita fue explosiva. Entre 1895 y 1930 se multiplicó por 187 veces, aunque su cobertura solo comprendía el 2,7\% de la cohorte de edad (AE, 1930). Los liceos femeninos no estudiaban educación cívica sino economía doméstica, y tanto en historia como en lengua castellana, el programa era el mismo de los liceos masculinos (Serrano, Ponce de León, \& Rengifo, 2012, p. 388).

La relación entre la escuela y la nacionalización del pueblo mapuche, que mantuvo su territorio independiente hasta la ocupación chilena en 1883 , ha sido difícil de investigar. El estado no tuvo una política educacional indígena. Fue una tarea que le encargo a misioneros franciscanos, capuchinos italianos y bávaros, y que asumieron también misioneros protestantes. La premisa estatal era que la escuela terminaría por asimilarlos al país. Pero no eran tan sencillo que llegaran a la escuela porque ella no tenía sentido en la cultura mapuche. De hecho, en el periodo hispano los caciques se negaron a enviar a sus hijos al Colegio de Naturales fundado en el siglo XVIII (Pinto, 1988) y apenas asistieron a las escuelas misionales hasta después de la ocupación en que el aprendizaje del idioma y de la escritura fueron destrezas útiles en la lucha jurídica por defender sus tierras. La escuela misional incorporo la dimensión étnica, era de hecho bilingüe tanto en la enseñanza como en los textos en su mayoría religiosos. En cambio la fiscal sencillamente la ignoro tratando a niños y niñas mapuches como pobres rurales y prohibiendo el uso de su lengua. No se sabe, en el periodo aquí tratado, cuantos mapuches fueron a la escuela porque en la misión había niños chilenos y en menor grado, en las fiscales había niños mapuches. Datos fragmentados muestran, por ejemplo, que una de las principales escuelas misionales tuvo en la década del 20 un promedio de tres quintos niños mapuches (ADV, Crónica Misión Padre Las Casas, 1935). ${ }^{4}$ Las escuelas misionales fueron para niñas y niños, fueron alfabetizadoras, evangelizadoras y prepararon para oficios manuales según género. La educación por sí misma era un elemento aculturador y en este sentido, nacionalizador, sin embargo ello no fue exitoso si se considera que la cultura mapuche ha tenido una gran capacidad de resistencia y de cambio. Las primeras generaciones de mapuches letrados formaron las organizaciones reivindicativas de su pueblo transformándose en un actor del escenario político nacional.

En síntesis, esta somera mirada permite atisbar cuan heterogénea eran los estudiantes, la enseñanza de la historia. Heterogéneas entre ricos y pobres, entre ciudad y campo, entre hombres y mujeres, entre mapuches y chilenos. Dicha heterogeneidad no niega la creación de un sentimiento de pertenencia nacional en la escuela, sino que lo historiza.

\section{Las batallas políticas}

El estudio de los textos escolares ha sido un campo importante en la historia de la enseñanza de la historia. Fuentes riquísimas para estudiar la hegemonía intelectual y política y sus conflictos, deben utilizarse críticamente si de ellos se quiere inferir lo que 
enseñaron los profesores y, más aun, aquello que procesaron los alumnos (Ossenbach \& Somoza, 2000). Estudiosos de textos escolares han llamado la atención sobre aquello, no obstante los expertos consideran ya demostrado que el currículo y los textos de historia suelen ser los principales medios de influencia, sino de control, sobre como los niños entienden el pasado de sus naciones (Foster, 2012; Romero, 2004). Al mismo tiempo, estudios de experiencias actuales, por ejemplo (Létourneau \& Moisan, 2012), muestran que la narrativa de los jóvenes sobre la historia de Quebec no es coincidente o semejante a los programas y textos, sino a la enseñanza de los profesores, que era convencional por presión y falta de instrumentos didácticos.

De todas formas, los textos de historia son fuentes para estudiar una narrativa histórica que forma parte de la narrativa política. En el caso chileno, como en la mayoría del continente, los textos muestran con transparencia los grandes debates políticos y no pretenden ocultarlos. En el relato compartido, el hito fundante de la nación era la Independencia (1810). El Imperio español había traído la civilización a la vez que la sujeción, aquella que la Independencia revirtió transformando el país en una "excepción" en América Latina pues había resuelto tempranamente las luchas internas, había consolidado el Estado y sus instituciones. Por ese motivo Chile había vencido a sus vecinos en dos guerras (1837 y 1879). Si para los conservadores ello fue posible gracias a la unión de la Iglesia y el Estado, para los liberales se debía a la progresiva emancipación de la Iglesia y a la gradualidad con que se había transitado desde una república conservadora a una liberal. La republica era finalmente, una construcción liberal y esa fue la interpretación hegemónica al finalizar el siglo. Los textos de educación cívica, algunos específicos orientados para los sectores populares, más tardíos, también resaltaron el carácter liberal de la organización jurídica del estado. ${ }^{5}$ Paralelamente aparecen textos de historia de Chile que levantaron una interpretación social demócrata, anti oligárquica, que atribuían a la educación pública el paso de una república liberal a una democrática. Era una narrativa construida principalmente en las cátedras de historia del Instituto Pedagógico de la Universidad de Chile que formaba a los profesores secundarios. A ella se le opuso por primera vez una visión nacionalista de carácter autoritario. Los textos de historia reflejan tres momentos y tres interpretaciones: uno liberal-republicano, centrado en el estado de derecho, las libertades y el sentido patriótico del servicio público; un segundo que extiende el vocabulario del patriotismo desde la esfera política a la social, denunciando la injusticia como incompatible con la ciudadanía y la democracia; y una tercera, propiamente nacionalista, en que el patriotismo se identifica con la unidad espiritual de la nación encarnada por el Estado. Esta última estuvo en tensión con la social demócrata dentro del establishment educativo. Durante la dictadura militar entre 1926 y 1931 el triunfo del discurso nacionalista fue evidente así como lo fue el discurso pedagógico democrático y social en los años 30 junto a la formación del Frente Popular. Estos textos son interpretativos y comprometidos ideológicamente, y no temen acercarse al tiempo presente. Esto cambia alrededor de la década del 40 en que los textos pasaron a ser secos y asépticos, una colección de datos a memorizar como si se optara por silenciar la conflictividad de la historia en una sociedad altamente fragmentada en lo social y de gran movilización política y pluralismo ideológico. ${ }^{6} \mathrm{El}$ programa oficial de 
historia de 1952 muestra el eclecticismo, si puede llamarse así, envuelto en la enseñanza de la historia desde la perspectiva estatal. Es francamente hispanista, Cristóbal Colón es el primer héroe y el segundo es Pedro de Valdivia, el conquistador del territorio que sería Chile. Es francamente liberal en lo que se refiere a la historia republicana, y los rasgos nacionalistas aparecen en "Chile Contemporáneo" cuya finalidad, señala, es "exaltar entre los alumnos el sentimiento patrio, de suerte que se forme en ellos un verdadero culto por los Padres de la Patria y por todos aquellos ciudadanos eminentes que nos dieron libertad y organización" (Republica de Chile, 1952). Es un periodo en que el lenguaje nacionalista impregna la economía, la cultura, la política siempre con el objetivo de profundizar la democracia a través de la movilización "patriótica” en pos del progreso económico a través de la industrialización, la crítica a la inversión extranjera, la necesidad de defenderse en un orden internacional y económico en que países pequeños y pobres eran muy vulnerables. Es un discurso que en otras partes del continente fue de la mano con el populismo. En Chile fue más débil por la fortaleza de los partidos políticos (Scully, 1992).

A nuestro juicio, el componente nacional no estuvo tanto en la enseñanza de la historia de Chile, que siguió siendo muy minoritaria en el currículo, sino en la transversalidad del mismo. Relacionado con lo anterior, se elabora una política explicita a partir de los años '30 para crear una "cultura nacional" que incorporase lo contemporáneo y rescatase la cultura popular tradicional. El gobierno a través del currículo estableció un canon de qué comprendía por ambas cosas. Canon muy discutible sobre su veracidad, pero no es ello lo que interesa aquí, cuanto que en los silabarios, libros de lectura, clases de gimnasia, educación musical aparecieron juegos tradicionales, liras y payas, comidas, cantos, bailes y paisajes de las distintas regiones así como las estereotipadas figuras de lo que se presentó como el prototipo nacional popular: el "huaso" campesino y el "roto" urbano. La enseñanza de la literatura, que comprendía textos clásicos europeos y especialmente españoles del siglo de oro así como literatura contemporánea francesa, norteamericana y latinoamericana, incorpora la "literatura chilena." En el siglo XIX era difícil construir un canon por su franca escasez, pero en el XX, y junto a la emergencia de los sectores medios letrados hijos del liceo y de la escuela, surgió efectivamente una literatura naturalista y realista cuya versión local tomo el nombre el criollismo y de novela social. Este "canon" era progresista, de fuerte contenido anti-oligárquico que denunciaba la miseria obrera y campesina. En general los cánones literarios nacionales solo admitían clásicos de la lengua vernácula. Balzac ingreso a los manuales de literatura francesa en 1880, treinta años después de su muerte, y al currículo en 1942 (Milo, 1997, p. 285). En Chile, donde los clásicos de la lengua pertenecen a una amplia área cultural, los autores nacionales entraban al currículo "recién salidos del horno." Poblaban las revistas de los liceos y las academias escolares que florecen en esos años. Es en la literatura donde la identificación de lo nacional adquiere una mayor densidad. Era una creación cultural nueva que dialogaba con distintos movimientos literarios internacionales y que buscaba su originalidad en la realidad chilena. El canon en 1930 era masculino y la primera mujer novelista entro en 1950.

El canon tuvo su momento de gloria y pudo construir el símbolo perfecto de 
lo que era la "cultura nacional." Una modesta preceptora primaria de una pequeña escuela rural obtuvo el Premio Nobel de Literatura en 1945. Sus versos se recitaban en las escuelas y cuando visito el país, miles de niños se las recitaron en las calles por las cuales paso su convoy. Gabriela Mistral, demócrata, anti nacionalista, pacifista, indigenista y latinoamericanista fue el símbolo de la educación y de la democracia chilena.

\section{Los ritos cívicos}

Los ritos cívicos han sido considerados como uno de los instrumentos más eficaces en la formación del nacionalismo y uno de los principales mecanismos de control del Estado en la creación de una adhesión y lealtad hacia la nación. En efecto, la literatura sobre la Revolución Francesa ha mostrado su enorme capacidad simbólica y movilizadora (Agulhon, 1979; Bell, 2003; Ozouf, 1976) así como en las Independencias hispanoamericanas (Annino, Castro, \& Guerra, 1994; Murillo de Carvalho, 1997). La escuela ha sido un espacio privilegiado para su reproducción. No obstante, como se ha intentado mostrar en los temas anteriores, requiere ser historizado y, por lo mismo, complejizado. En el caso que tratamos, representativo de muchas experiencias no europeas, los rituales cívicos en los establecimientos escolares fueron tardíos, desiguales entre la primaria y la secundaria, entre el campo y la ciudad, entre la elite, los sectores medios y populares.

La ruralidad fue un muro impenetrable para la escuela moderna. La ruralidad americana nada tiene que con la Europea porque una se da en territorios gigantes con escasa población y la otra en pequeñas villas. En 1930 la densidad de la población europea era de 46,3 habitantes y la de América era de 4,9 (Labarca, 2011, p. 37). La población rural chilena vivía diseminada en el campo profundo, en la hacienda principalmente, y fue la mayoría hasta la década del 30. Era difícil que la escuela llegara a esos niños, pero fue llegando lentamente con escuelas mixtas, escuelas volantes, escuelas de tercera categoría, como ya se mencionó. La mayoría de las escuelas urbanas y rurales del XIX eran una pieza arrendada o un rancho con una empalizada, sin vidrios, con algunos troncos como asientos y escasos escritorios. La escuela era una institución "sin domicilio" que se mudaba según el movimiento de la población. La escuela, en realidad, era el preceptor.

El gobierno distribuyo útiles escolares, textos, pizarras, tizas, mando los modelos de escritorios y bancos para que fueran replicados por los carpinteros locales. Procuro enviar mapas. Primero mapa mundi comprados en Boston y luego láminas con mapas de Chile. En los inventarios del archivo del Ministerio no figura el envío de una sola bandera. Los detallados relatos de los visitadores de escuelas no mencionan que se cante el himno nacional, ni que se celebren efemérides. Se celebra el aniversario de la Independencia porque era una fiesta antigua y popular. A comienzos del XX, fruto de la riqueza del salitre, la escuela encuentra su propia arquitectura en las ciudades y villas, preferentemente en la plaza, y en ella flamea la bandera y brilla el escudo en su frontis. Los ritos cívicos se multiplican tanto en la ciudad como en los establecimientos escolares. Los estudiantes participan en ambas, son el "voluntariado" perfecto para llenar la plaza y desfilar por las calles. Estas celebraciones tienen un formato militar, 
frecuentemente organizadas desde guarniciones y regimientos, donde participan todas las autoridades locales, entre ellas el director del liceo y de la escuela principal (Rojas, 2004). El gobierno obliga a replicarlos y multiplicarlos dentro de los recintos escolares femeninos y masculinos a través de actos, versos, composiciones, disertaciones, dibujos referidos a la patria y a los héroes.

Todo ello es llamado "chilenidad" que hacia los 40, coherente con lo antes señalado, adquirió un carácter más cultural que militar (De la Chilenidad, 1950). Es posible que para entonces todos los niños que iban a la escuela supieran que eran chilenos, aunque no sabemos qué significado tendría para ellos. Sin embargo, estas actividades eran minoritarias en relación a las muchísimas que realizan los establecimientos. En las actas de los consejos de profesores de los liceos, por ejemplo, tantos en los masculinos como en los femeninos que se han encontrado, los preparativos de las fiestas cívicas apenas se mencionan, solo se notifican, en cambio la preparación de las actividades propias ocupan más espacio incluso que las materias académicas o administrativas. A ellas destinan sus escasos fondos y organizan fiestas para recaudar recursos. Por mucho, la conmemoración más importante era el aniversario del liceo. Tanto así que el Ministerio de Educación pidió austeridad sin éxito alguno. Eran tres días de fiesta en que participaba activamente la comunidad local. Se cantaba la canción nacional, pero a continuación el himno del colegio, una novedad que se agrega a los discursos sobre su historia, sobre sus antiguos profesores; los padres de familia y las asociaciones de ex alumnos, dos actores nuevos, otorgan premios y ponen placas recordatorias. Y hay muchos más actos: día del americanismo, de la salud, del ahorro, del niño, del maestro, actividades recreativas y culturales, premiaciones, bienvenidas, despedidas. Las competencias deportivas adquirieron enorme relevancia porque se competía con liceos de ciudades vecinas, incluso en la capital, el Instituto Nacional competía con colegios particulares de elite, una competencia más que simbólica. Los liceos masculinos y femeninos por igual - aunque ellos jugaban fútbol y ellas basquetbol - movilizaban a todos los cursos con gran entusiasmo. Los liceos adquieren los nombres de figuras educacionistas nacionales o locales propuestos por ellos. Las revistas escolares, aunque algunas fueran de corta vida, tratan principalmente la inserción del liceo en la comunidad y las actividades de los centros de alumnos, otra institución nueva, dentro y fuera del establecimiento.

La "identidad comunitaria escolar" y la "identidad comunitaria local" aparecen con más fuerza que la nacional, con más vida, más espontaneidad, más participación, más sentido. Los liceos van construyendo una representación de sí mismos como el faro de la comunidad, como su emblema, como la promesa de su futuro progreso. Es desde la identidad local que se establece el vínculo con la pertenencia nacional. Los establecimientos forman parte, y sus miembros lo saben, de una gran red de educación pública, dependen del Estado central y se auto representan como un aporte a la patria. ${ }^{7}$ Ello resulta concordante con investigaciones tan diversas como las de Chavel (1996) en las escuelas francesas entre fines del siglo XIX y comienzos del XX o las de Vaughan (2000) en las comunidades indígenas en México en la década de 1940. La educación, entonces, como forjadora de una identidad nacional resulta abstracta, desde arriba hacia abajo, si no se considera esta nueva identidad que es la 
propiamente escolar y su inserción local.

\section{Consideraciones finales}

Este artículo ha querido proponer algunos problemas para historizar un supuesto como es la relación entre educación nacional, enseñanza de la historia, ritos cívicos y formación de las identidades nacionales y los nacionalismos. Si se busca rescatar a los actores sociales, si se puede avanzar desde el estudio de una política estatal a la de las prácticas escolares, considerando las enormes dificultades metodológicas que ello implica, preguntas básicas como las aquí propuestas pueden contribuir en esa dirección. Las preguntas pueden ser válidas para cualquiera investigación sobre el tema. Los hallazgos son particulares y por ello permiten poner a prueba los supuestos y las relaciones de causalidad. Plantea el problema de cuan distintos pueden haber sido los nacionalismos en distintas regiones, especialmente con respecto Europa. Este artículo muestra que la enseñanza de la historia tuvo primero un fin ejemplarizante y más tarde histórico y nacional; que la historia nacional tuvo un espacio muy menor dentro de la enseñanza de la historia en relación a la europea; que la literatura fue más significativa en la formación de una pertenencia nacional que la historia. La formación de un sentimiento o de una conciencia de pertenencia nacional es un proceso desigual y para nada lineal según clase social, genero, etnia, asentamiento territorial, demografía, y el ritmo es distinto según la cobertura escolar. Intentar estudiar la relación antes aludida desde su recepción y apropiación requiere buscar nuevas fuentes que en este caso han sido los archivos de los propios establecimientos escolares. Ello ha permitido resaltar la importancia de la identidad comunitaria escolar y local sin las cuales es difícil comprender la identidad nacional que busca imponer el estado. Por último, estimamos que este es un debate que no concierne solo a la historiografía y a la historia de la educación, sino también a la didáctica de la historia.

\section{Notas}

1. Por "lo nacional” se entenderá aquí cualquier tipo de vínculo, en cualquier formato, que aluda a la organización política llamada por los actores con el nombre de nación, patria, estado nacional o el nombre propio de país.

2. Memoria del Ministerio de Justicia, Culto e Instrucción Pública.

3. Anuario Estadístico.

4. Archivo Diócesis de Villarrica.

5. Los más representativos son Suarez, J. B. (1882). Catecismo constitucional de la República de Chile, extractado del "Manual del Ciudadano" aprobado por la Universidad para la enseñanza en los colejios $i$ destinado a la clase obrera $i$ a los alumnos de las escuelas primarias. Santiago, Chile: Imprenta de El Correo; Concha, M. (1909). Cartilla de Educación Cívica: elementos de Derecho Público y de Economía Política. Santiago, Chile: Imprenta Cervantes.

6. Un ejemplo del primero es Domingo Amunátegui Solar, Historia de Chile. Texto 
Aprobado por el Ministerio de Educación. T.I correspondiente a V de Humanidades, T.II correspondiente a VI humanidades; Ed. Nacimiento, Santiago, 1933. Un ejemplo del segundo es Francisco Díaz Valenzuela, Historia y Geografía, Ed. Nacimiento, Santiago, 1936.

7. Lo anterior está basado en los Archivos del Instituto Nacional, Liceo 1 Javiera Carrera de niñas de Santiago, Liceo de Talca (masculino), Liceo Rebeca Charme de Talca (femenino), Liceo de San Fernando (masculino) y Archivo de la Prefectura Apostólica de la Araucanía, Diócesis de Villarrica, Chile.

\section{Referencias}

Agulhon, M. (1979). Marianne au combat: L'Imagerie et la symbolique républicaines de 1789 a 1880. Paris, France: Flammarion.

Anderson, B. (1993). Comunidades imaginadas. Reflexiones sobre el origen y la difusión del nacionalismo. Mexico City, Mexico: Fondo de Cultura Económica.

Annino, A., Castro, L., \& Guerra, F.-X. (1994). De los imperios a las naciones: Iberoamérica. Zaragoza, Spain: IberCaja.

Barton, K. (2012). School history as a resource for constructing identities: Implications of research from the United States, Northern Ireland and New Zealand. In M. Carretero, M. Asensio, \& M. Rodríguez-Moneo (Eds.), History education and the construction of national identities (pp. 93-107). Charlotte, NC: Information Age Publishing.

Bell, D. (2003). The cult of the nation in France: Inventing nationalism, 1680-1800. Cambridge, MA: Harvard University Press.

Carretero, M., \& López, C. (2012). Commentary: Identity construction and the goals of history education. In M.Carretero, M. Asensio, \& M. Rodríguez-Moneo (Eds.), History education and the contruction of national identities (pp. 139-150). Charlotte, NC: Information Age Publishing.

Chavel, J.-F. (1996). L'École republicaine et les Petites Patries. Paris, France: Autiers.

Cox, C., \& Gysling, J. (2009). La formación del profesorado en Chile 1842-1987. Santiago, Chile: Ediciones Diego Portales.

De la Chilenidad. (1950). Circular 3 de la Dirección General de Educación Primaria. Osorno, Chile: Imprenta Cervantes.

De La Taille, A. (2012). Educar a la Francesa: Anna du Rousier y el impacto del Sagrado Corazón en la mujer cbilena. Santiago, Chile: Ediciones Universidad Católia de Chile.

Fendler, L. (2013). There are no independent variables in history. In T. Popkewitz (Ed.), Rethinking the history of education: Transnational perspectives on its questions, methods, and knowledge (pp. 264-291). New York, NY: Palgrave.

Foster, S. (2012). Re-thinking history textooks in a globalized word. In M. Carretero, M. Asensio, \& M. Rodríguez-Moneo (Eds.), History education and the construction of national identities (pp. 49-62). Charlotte, NC: Information Age Publishing.

Garcia, P., \& Leduc, J. (2004). L'enseignement de l'histoire en France. Paris, France: Armand Colin. 
Gazmuri, C. (2006), La historiografía chilena (1842-1970). Santiago, Chile: Taurus.

Gellner, E. (1983). Nations and nationalism. Oxford, England: Basil Blackwell.

Gobat, M. (2013). The invention of Latin America: A transnational history of anti-imperialism, democracy, and race. American Historical Review, 118, 1345-1375. http://dx.doi. org/10.1093/ahr/118.5.1345

Gonzalbo, P. A. (2002). Historia y nación: I. Historia de la educación y enseñanza de la bistoria. Mexico City, Mexico: El Colegio de México.

Herrera, M.C. (2007). Political culture, school texts and Latin American societies: Introduction. Paedagogica Historica: International Journal of the History of Education, 43(5), 627-631. http://dx.doi.org/10.1080/00309230701587116

Hobsbawm, E. J. (1991). Naciones y nacionalismos desde 1780. Barcelona, Spain: Editorial Crítica.

Labarca, A. (2011). Bases para una política educacional. Santiago, Chile: Cámara Chilena de la Construcción.

Létourneau, J., \& Moisan, S. (2012). Young people's assimilation of a collective historical memory: A case study of Quebeckers of French-Canadian heritage. In P. Seixas (Ed.), Theorizing historical consciousness (pp. 109-128). Toronto, ON: University of Toronto Press.

Lowe, R. (1999). Education and national identity. History of Education, 28(3), 231-233. http://dx.doi.org/10.1080/004676099284591

Michelet, J. (1860). Compendio de la historia moderna. Santiago, Chile: Imprenta del Ferrocarril.

Michelet, J. (1897). Précis de l'bistoire moderne. Introduction a l'bistoire universelle. Paris, France: Ernest Flammarion.

Milo, D. (1997). Les classiques scolaires. In P. Nora (Ed.), Les lieux de mémoire. La republique, la nation, les Francais. Paris, France: Quarto Gallimard.

Murilo de Carvalho, J. (1997). La formación de las almas: El imaginarios de la república en el Brasil. Buenos Aires, Argentina: Universidad Nacional de Quilmes.

Nora, P. (1997). Les lieux de mémoire. La republique, la nation, les Francais. Paris, France: Quarto Gallimard.

Ossenbach, G., \& Somoza Rodríguez, J. M. (2000). Los manuales escolares como fuente para la bistoria de la educación en América Latina. Madrid, Spain: UNED.

Ozouf, M. (1976). La fete révolutionare 1789-1799. Paris, France: Editions Gallimard.

Pinto, J. (1988). Misioneros en la Araucanía 1600-1900. Temuco, Chile: Editorial Universidad de la Frontera.

Pozo Andrés, M. (2008). Presentación: Educación y construcción de las identidades nacionales. Historia de la Educación, 27, 27-36.

Republica de Chile. (1952). Prigrama de estudio del primer cclo de bumanidades. Santiago, Chile: Imprenta Nacimiento.

Riekenberg, M. (1991). Latinoamérica: Enseñanza de la historia, libros de textos y conciencia bistórica. Buenos Aires, Argentina: Alianza Editorial.

Rojas, J. (2004). Moral y prácticas cívicas en los niños Chilenos, 1880-1950. Santiago, Chile: Ariadna Ediciones.

Sarmiento, D. F. (1845). Civilización i barbarie: vida de Juan Facundo Quiroga i aspecto fisico, costumbres $i$ habitos de la Republica Argentina. Santiago, Chile: Imprenta del Progreso.

Scully, T. (1992). Rethinking the center: Party politics in nineteenth and twentieth century Chile. 
Stanford, CA: Stanford University Press.

Seixas, P. (2012). Theorizing historical consciousness. Toronto, ON: University of Toronto Press.

Serrano, S., Ponce de León, M., \& Rengifo, F. (Eds.). (2012). Historia de la educacion en Chile (1810-2010, Vol. 1). Santiago, Chile: Taurus.

Serrano, S., Ponce de León., M., \& Rengifo, F. (Eds.). (2012). Historia de la educación en Chile (1810-2010, Vol. 2). Santiago, Chile: Taurus.

Svein, O. (2001). Educational ideas and nation building in Norway 1840-1900. In S.

Ahonen \& J. Rantala (Eds.), Nordic lights: Education for nation and civic society in the Nordic countries, 1850-2000. Helsinki, Finland: Finnish Literature Society.

Symcox, L., \& Wilschut, A. (2009). National history standards: The problems of the canon and the future of teaching history. Charlotte, NC: Information Age Publishing.

Tanck, D. (2011). Historia mínima ilustrada: La educación en México. Mexico City, Mexico: El Colegio de México.

Vásquez, J. Z. (1991). Textos de historia al servicio del nacionalismo. In M. Riekenberg (Ed.), Latinoamérica: Enseñamza de la historia, libros de textos y conciencia histórica. Buenos Aires, Argentina: Alianza Editorial.

Vaughan, M. K. (2000). La politica cultural de la revolución: Maestros, campesinos y escuelas en México, 1930-1940. Mexico City, Mexico: Fondo de Cultura Económica.

Wilschut, A. (2012). Images of time: The role of bistorical consciousness of time in learning bistory. Charlotte, NC: Information Age Publishing. 